\title{
Use of Peptide Arrays for Identification and Characterization of
}

\section{LIR Motifs}

Mads Skytte Rasmussen, Åsa Birna Birgisdottir and Terje Johansen

Molecular Cancer Research Group, Department of Medical Biology, University of Troms $\varnothing-$ The Arctic University of Norway, 9037 Troms $\varnothing$, Norway.

Corresponding author Terje Johansen; E-mail: terje.johansen@uit.no)@uit.no

Running Head: Mapping of LIR motifs using peptide arrays 


\begin{abstract}
The mammalian ATG8 proteins (LC3A-C/GABARAP, GABARAPL1 and -L2) are small ubiquitin-like proteins critically involved in macroautophagy. Their processed C-termini are post-translationally, conjugated to a phosphatidylethanolamine moiety, enabling their insertion into the lipid bilayers of both the inner and outer membranes of the forming autophagosomes. The ATG8s bind a diverse selection of proteins including cargo receptors for selective autophagy, members of the core autophagy machinery and other proteins involved in formation, transport and maturation (fusion to lysosomes) of autophagosomes. Protein binding to the ATG8s is in most cases mediated by short, conserved sequence motifs known as LC3-interacting regions (LIRs). Here, we present a protocol for identifying putative LIR motifs in a whole protein sequence using peptide arrays generated by SPOT synthesis on nitrocellulose membranes. The use of two-dimensional peptide arrays allows for further identification of specific residues critical for LIR binding.
\end{abstract}

Key words Autophagy, Atg8, GABARAP, LC3, LIR, Peptide array 


\section{$1 \quad$ Introduction}

Autophagy processes, whereby cytoplasmic content is degraded in the lysosome, consist of at least three different main pathways including macroautophagy, microautophagy and chaperone-mediated autophagy [1]. Macroautophagy (for simplicity hereafter referred to as autophagy) can be subdivided into selective autophagy or unspecific, bulk autophagy. Selective autophagy allows for targeted degradation of large substrates (damaged or surplus mitochondria, peroxisomes or ER and protein aggregates as wells as invading bacteria and viral capsids), but also of specific signaling proteins, as well as recycling of metabolic substrates (lipids and glycogen)[2-5]. Selective autophagy receptors are able to simultaneously bind the cargo and ATG8 family proteins attached to the inner membrane of the forming autophagosome $[2,6]$. Cargo recognition is often mediated through specific ubiquitin tags on the substrates that are recognized by the SLR (sequestosome 1-like receptors) family of cargo receptors which includes p62/SQSTM1(sequestosome 1)[6], NBR1 [7], OPTN [8], NDP52 [9], Tax1BP1 [10], and yeast Cue5 [11]. NDP52 is also indirectly recruited to cargo by recognizing Galectin-8, a cytosolic lectin, acting as a danger receptor that detects bacterial invasion by binding host glycans exposed on damaged pathogencontaining vacuoles or sterile damage to lysosomal or endosomal membranes [12]. Some autophagy receptors may directly recognize cargo as exemplified by TRIM family E3 ligases acting as cargo receptors for i.e. viral capsids and recruitment hubs for autophagy components [13]. Direct recognition is also mediated by Atg19 and Atg34, acting in the biosynthetic cytoplasm to vacuole targeting (Cvt) pathway in yeast, which mechanistically is similar to autophagy [14, 15]. NCOA4 directly targets surplus ferritin for autophagic degradation [16]. Autophagy receptors may also be present on the cargo itself because they reside in the outer membranes of organelles like NIX/BNIP3L [17], FUNDC1 [18], and FKBP8 [19], in mitochondria, and FAM134B in ER [20]. 
All cargo receptors identified so far contain an LC3-interacting region (LIR) [21], also referred to as AIM (Atg8-family interacting motif) in yeast [22]. Canonical LIR sequences consist of a short linear motif of up to 13 amino acids with a five-amino acid long core sequence following the consensus $\Theta-\mathrm{X}-\mathrm{X}-\Gamma-$, where $\Theta$ is an aromatic amino acid $(\mathrm{W} / \mathrm{F} / \mathrm{Y})$, $\Gamma$ is a hydrophobic amino acid (L/I/V), and X can be any amino acid [21]. The LIR allows for tight association of the cargo receptor with the ATG8s (LC3s/GABARAPs) through interaction with two hydrophobic pockets on the ATG8s [21,22]. The ATG8s are small ubiquitin-like proteins covalently attached to the lipid phosphatidylethanolamine (PE) in the autophagosomal membrane and function as anchoring points for the cargo receptors at the autophagosome [23]. Thus, the LIR is crucial for the ability of the cargo receptors to guide their target to degradation by autophagy. In addition to autophagy receptors, ATG8 proteins also bind a diverse selection of LIR-containing proteins including members of the core autophagy machinery [24], proteins involved in transport of autophagosomes[25-27], crosstalk with endocytosis [28], and fusion of autophagosomes with lysosomes [29]. The ability of ATG8 proteins to scaffold proteins and protein complexes on membranes via LIRdependent interactions is not limited to autophagy but also observed in spatially restricted cellular signaling [30].

Canonical LIR sequences can be predicted by use of iLIR, a web-based application [31], or by manually searching the sequence of a protein for LIR-like motifs. However, LIR-like sequences can often be identified in most proteins and require experimental testing for validation. Relying only on linear sequence motifs resembling LIR-like motifs might also result in false negatives as a growing number of proteins have been shown to utilize noncanonical LIRs such as NDP52 [32], Bcl-2 [33], Atg12 [34] and UBA5 [35]. These non- 
canonical LIRs may lack the conserved aromatic residue, like the C-LIR of NDP52 [32], or the conserved hydrophobic residue, like the LIR of Bcl-2 [33]. In UBA5 the spacing between the conserved aromatic and hydrophobic residue is only one residue and another hydrophobic residue spaced 3 amino acids from the aromatic residue is also important for binding [35]. In ATG12 the aromatic and hydrophobic residue are spaced far apart in the primary sequence, but brought together in a three-dimensional binding epitope [34]. Furthermore, we are as of yet not able to predict the precise determinants for selective binding to the different ATG8s, as some only bind GABARAPs while others only bind LC3s and some bind all ATG8s with high affinity $[21,36]$.

In this chapter, we provide a protocol for mapping LIRs in ATG8 interacting proteins by use of peptide arrays produced by SPOT synthesis on nitrocellulose membranes. Consisting of small linear peptides up to about 20 residues, the peptide array is well suited to probe for possible LIRs in the target protein as well as revealing the importance of single residues of the LIR motif for ATG8 binding. To search a protein for candidate LIR motifs, an array of 20mer peptides covering the entire sequence of the target protein is synthesized and immobilized on a cellulose membrane using SPOT synthesis $[37,38]$. For routine identification of LIR motifs, the membrane is synthesized with 20-mer peptides starting at the $\mathrm{N}$-terminal end of the protein sequence moving the overlapping peptides 3 residues towards the $\mathrm{C}$-terminal end in each spot relative to the peptide in the previous spot. The membrane is then probed with recombinant GST or GST-LC3s/GABARAPs after which binding is detected with an antiGST antibody (Fig. 1). Care should be taken in choosing an appropriate ATG8, as several LIR-containing proteins only bind strongly to either GABARAPs [24, 36, 39], or LC3A/B $[18,26]$. Once a LIR motif has been identified, the individual LIRs can also be probed for specificity against the different ATG8s by performing single spot analysis of 20-mers 
containing the LIR motif followed by separate probing with either GABARAP or LC3B [40] (Fig. 2). Two dimensional peptide arrays consisting of an 18-mer containing the LIR motif can be used to probe the residue requirements of the LIR motifs by substituting each position of the sequence with all of the 19 alternative amino acids [26] (Fig. 3). The results obtained by peptide arrays should always be confirmed with other methods, such as GST-pulldowns or immunoprecipitations with the full-length proteins with WT and mutated LIR sequences, or other in vitro and/or in vivo protein-protein interaction assays.

\section{Materials}

\subsection{Synthesis of Peptide Arrays}

Synthesis of peptide arrays on cellulose membranes were performed using a MultiPep automated peptide synthesizer (INTAVIS Bioanalytical Instruments AG, Germany). Membranes were ordered from Ola Rumohr Blingsmo at the Centre for Molecular Medicine Norway, NCMM - Administration and Core Facilities (NCMM ADMIN), Faculty of Medicine, University of Oslo. Design of the arrays was done using MultiPep peptide-synthesis control software (INTAVIS AG, Germany) version 1.5.06.

\subsection{Production and Purification of GST-Atg8/LC3/GABARAPS}

1. GST expression vector e.g. pDest15 (Invitrogen, 11802014).

2. Escherichia coli suitable for protein production e.g. BL21 STAR (DE3) pLysS cells (Thermo Fisher, C602003).

3, 2x YT medium: [16\% Bacto-tryptone (w/v), 10\% yeast-extract (w/v), 5\% $\mathrm{NaCl}(\mathrm{w} / \mathrm{v})]$ with ampicillin $(100 \mu \mathrm{g} / \mathrm{mL})$. 
4. Suitable centrifuge e.g. JLA 16.250 fixed angle rotor (Beckman Coulter).

5. Lysis buffer: $50 \mathrm{mM}$ Tris- $\mathrm{HCl} \mathrm{pH} 8.0,250 \mathrm{mM} \mathrm{NaCl}, 1 \mathrm{mM}$ DTT, $0.35 \mathrm{mg} / \mathrm{mL}$ lysozyme.

6. Sonicator e.g. VCX 130 Sonic Vibra-Cell ultrasonic processor.

7. GST-binding column e.g. GSTrap ${ }^{\mathrm{TM}} \mathrm{FF} 1 \mathrm{~mL}$; GE Healthcare.

8. Elution buffer: $1 \times$ PBS $\mathrm{pH} 8.0,1 \mathrm{mM}$ reduced glutathione, the buffer is $\mathrm{pH}$ adjusted, sterile filtered, and degassed before use.

9. Chromatography system e.g. ÄKTAprime chromatography system; GE Healthcare.

10. Bradford protein assay or similar.

\subsection{Peptide Overlay Assay}

1. Ponceau S Staining Solution: 0.1\%(w/v) Ponceau S (Sigma-Aldrich, \# P3504) in $5 \%(\mathrm{v} / \mathrm{v})$ acetic acid.

2. TBS-T buffer: $10 \mathrm{mM}$ Tris- $\mathrm{HCl} \mathrm{pH} 7.4,150 \mathrm{mM} \mathrm{NaCl}$, and 0.1\% Tween-20.

3. HRP conjugated GST-antibody clone RPN1236; GE Healthcare.

4. Western blotting Luminol Reagent kit (SC-2048, Santa Cruz Biotechnology).

5. Chemiluminescence capturing device e.g. charge-coupled device (CCD) camera or Xray film exposure.

6. Regeneration buffer for stripping of membrane: $62.5 \mathrm{mM}$ Tris- $\mathrm{HCl} \mathrm{pH}$ 6.7, $2 \%$ SDS and $70 \mu \mathrm{l}$ 2-mercaptoethanol per $10 \mathrm{ml}$ buffer. 


\section{Methods}

\subsection{Production and Purification of GST-Atg8/LC3/GABARAPS}

1. Subclone cDNAs encoding Atg8/LC3/GABARAPs in-frame with an N-terminal GST tag in an IPTG-inducible expression vector. Use the vector to transform a suitable strain of Escherichia coli, i.e. BL21 STAR (DE3) pLysS cells (Thermo Fisher).

2. Pick a single colony of transformed E. coli, transfer into a sterile tube with $5 \mathrm{~mL}$ of $2 \mathrm{x}$ YT medium, and incubate overnight at $37^{\circ} \mathrm{C}$ on a horizontal shaker $(250 \mathrm{rpm})$.

3. Dilute the overnight culture 1:20 to a final volume of $100 \mathrm{~mL}$ of $2 \mathrm{x}$ YT medium with ampicillin $(100 \mu \mathrm{g} / \mathrm{mL})$, and grow further under same conditions until the cells reach $\mathrm{OD}_{600}$ of 0.9 (about $2-3 \mathrm{~h}$ ).

4. Induce expression of the target gene by adding IPTG to a final concentration of $0.5 \mathrm{mM}$. Allow bacteria to grow for another $3-4 \mathrm{~h}$ at room temperature on a horizontal shaker.

5. After induction, transfer the bacterial culture into a sterile $250-\mathrm{mL}$ plastic flask and centrifuge at $3000 \mathrm{~g}$ at $4^{\circ} \mathrm{C}$ for $10 \mathrm{~min}$.

6. Remove the supernatant completely and resuspend the recovered pellet in $4 \mathrm{~mL}$ icecold lysis buffer. (see Note 1).

7. Keep the lysate on ice for $20 \mathrm{~min}$ and then add Triton $\mathrm{X}-100$ to $1 \%(\mathrm{v} / \mathrm{v})$.

8. Distribute the lysate into three tubes (about $1.5 \mathrm{~mL}$ per tube) (see Note 2).

10. Sonicate on ice (four times at $40 \%$ amplitude and $20 \mathrm{kHz}$ ). (see Note 3). 
11. Transfer to a microfuge tube and centrifuge at $15,000 \mathrm{~g}$ at $4{ }^{\circ} \mathrm{C}$ for $10 \mathrm{~min}$.

12. Filter the supernatant by passing through a $0.45-\mu \mathrm{m}$ sterile filter and apply on a GSTbinding column.

13. Elute proteins from the column with elution buffer at the speed of $1 \mathrm{~mL} / \mathrm{min}$ using a suitable chromatography system.

14. Use the Bradford protein assay to estimate protein concentration in the fractions where the protein is eluted.

\subsection{Set Up of Peptide Arrays for 20,3 or 18,1 Scanning and 2D Substitution Analyses}

1. Before doing a peptide array scan to identify putative LIR motifs in a protein it is advised to do GST pulldown assays or immunoprecipitations to check for a possible direct interaction between the protein of interest and ATG8 family proteins (see Note 4). The iLIR prediction server can also be used to look for consensus LIR motifs in the protein.

2. We have usually ordered the synthesis of two 540 grid membranes ( $30 \times 18$ peptides SPOT synthesized) to allow the search or scan for motifs binding to ATG8s in multiple proteins as this is the most cost effective way. This allows the analysis of on average about 2-5 proteins per membrane (4-10 proteins in total) depending on the length of each protein.

3. Each protein sequence is scanned in a 20,3 manner meaning that the membranes contain overlapping 20-mer peptides that are shifted with 3 amino acids per peptide starting at the N-terminal end of the protein. This usually also allows for quite accurate 
determination of the extent of the sequence motif required for efficient binding. If higher resolution is needed an 18,1 array can be used where the overlapping 18-mer peptides are shifted with only one amino acid per spot.

4. For 2D arrays we use 18-mer peptides and spot two rows of WT sequence at the top and then peptides with single substitutions of every amino acid at one position at the time from the N-terminal end of the 18-mer to the C-terminal end (see Fig. 3). We locate the core LIR motif (W/F/Y-X-X-L/I/V) in the middle of the peptide so we can look for residues both $\mathrm{N}$ - and $\mathrm{C}$-terminal residues to the core that may influence binding. However, some LIRs are located at the C-terminus of the protein in question, like human ATG4B, or yeast Atg19. We then, of course, locate the LIR at the end of the peptide as in its native protein.

\subsection{Peptide Overlay Assay}

1. Stain the peptide array membrane with Ponceau $\mathrm{S}$ for $1 \mathrm{~min}$.

2. Wash several times with water to allow visualization of peptide spots.

3. Dry the membrane overnight.

4. On the day of the assay, activate the membrane by incubating in $100 \%$ methanol for $10 \mathrm{~s}$.

5. Wash two times, at 5 min each, in TBS-T buffer.

6. Block nonspecific labeling by incubating the membrane in TBS-T containing 5\% $(\mathrm{w} / \mathrm{v})$ nonfat dry milk powder for $1 \mathrm{~h}$ at room temperature. 
7. To test binding to GST-Atg8/LC3/GABARAP, incubate the membrane with the purified GST-Atg8/LC3/GABARAP protein at 1-2 $\mu \mathrm{g} / \mathrm{mL}$ concentration in TBS-T buffer (the volume is adjusted according to the size of the membrane) for $2 \mathrm{~h}$ at room temperature. (see Note 4).

8. After binding, wash the membrane five times in TBS-T.

9. Incubate overnight at $4{ }^{\circ} \mathrm{C}$ with HRP-conjugated anti-GST at 1:5000 dilution in $5 \%$ dry milk.

10. Wash the membrane five times in TBS-T.

\subsection{Detection of Atg8/LC3/GABARAP Binding by Chemiluminescence}

1. Mix equal amounts of Luminol Reagent Solution A and Solution B (use $0.125 \mathrm{ml} / \mathrm{cm}^{2}$ total volume) by inversion in a screw cap vial.

2. Pour off the TBS-T wash solution from the membrane. Add the mixed Luminol Reagent Solutions to the membrane (peptide spot side facing up). Incubate for 1 minute at room temperature.

3. Lift the membrane with tweezers to drain excess Luminol Reagent and wrap the membrane (peptide spot side facing up) tightly in a single layer of plastic wrap.

4. Carefully place the plastic wrapped membrane (peptide spot side facing up) in a film cassette. In a darkroom, expose the membrane to film for $1 \mathrm{~min}$ and develop according to standard protocols for autoradiogram. Exposure time may be altered as necessary. 
5. Alternatively, place the plastic wrapped membrane in a Chemiluminescence capturing device e.g. charge-coupled device (CCD) camera for exposure and signal detection using the appropriate settings. (see Note 5).

\subsection{Analysis of Peptide Arrays}

1. The 20,3 or 18,1 scans are read off by scoring the peptide spots with a clear signal above background as positive. This allows the identification of positive peptides and the extent of the positive region to be mapped (see Fig. 1) (see Note 6).

2. For the 2D arrays the analysis of residues important for binding (see Fig. 3) is either done in a qualitative manner noting the loss of signal with certain substitutions, or the arrays can be analyzed by quantifying signal intensity from the chemiluminescent detection imaging device.

\subsection{Stripping of the Membrane}

1. In the event that no LIR is identified and one wants to reprobe the membrane with another ATG8 protein the membrane can be stripped and reprobed. If the membrane is dried out, it must be regenerated in methanol for some seconds.

2. Wash 3 times in water for 10 min each at room temperature.

3. Warm the regeneration buffer in a petri dish to $50^{\circ} \mathrm{C}$ and incubate the membrane for 10 minutes. Repeat twice.

4. Incubate 3 times in $10 \mathrm{x}$ TBS-T, for $10 \mathrm{~min}$ each, at room temperature.

5. Incubate 3 times in TBS-T, for $10 \mathrm{~min}$ each, at room temperature 
6. To check the success of the stripping procedure, block the membrane in $5 \%$ milk in TBS-T for $2 \mathrm{~h}$ at room temperature and incubate with HRP-conjugated anti-GST following the developing procedure as described above.

7. To reprobe the membrane with a new protein, wash the membrane five times, 4 min each, in TBS-T buffer and block the membrane in $5 \%$ milk in TBS-T buffer for $2 \mathrm{~h}$ at room temperature. Then incubate the membrane with the new GST-ATG8 protein following the peptide array procedure as described above.

\section{$4 \quad$ Notes}

1. Albeit the buffer itself can be stored at RT, lysozyme and DTT must be added prior to use.

2. Alternatively transfer to three cryotubes and store at $-70^{\circ} \mathrm{C}$. Allow for slow thawing of the E. coli lysates.

3. It is recommended to purify the GST-Atg8/LC3/GABARAP the same day or the day before the overlay assay. The purified protein can be kept at $4{ }^{\circ} \mathrm{C}$ for brief storage.

4. We have successfully used GST-fusions of yeast (S. cerevisiae) Atg8, Drosophila melanogaster Atg8a, human LC3A, LC3B, LC3C, GABARAP and GABARAPL1 to probe peptide arrays to identify LIR motifs. For human proteins our first choice is to use GST-GABARAP or GST-GABARAPL1 to probe the arrays with since they bind to most LIR-containing proteins and are therefore suitable for the first screening. However, if GST pulldowns of in vitro translated protein, or protein from cell extracts, or immunoprecipitations from cells, show a very strong preference for LC3A or -B, we use GST-LC3A or -B for the peptide array. 
5. The chemiluminescence method is a highly sensitive detection method and usually works well for the detection of LIR motifs within minutes with the described protocol. However, be observant to appearance of clear spots with an outer black ring around them. Such spots usually indicate that all the luminescence substrate has been used up (due to high secondary antibody binding). New substrate should then be added to the membrane followed by immediate detection with shorter detection time. Alternatively wash the membrane extensively in TBS-T and reapply chemiluminescence substrate.

6. Care should be taken when probing sequences enriched in hydrophobic regions containing multiple $\mathrm{W}$ or $\mathrm{F}$ residues such as i.e. WD40 or KELCH repeat proteins, as these regions might produce false positives if spaced in such a way that allows for docking into the HP1 and HP2 hydrophobic pockets of ATG8s.

Knowledge of the structure of the protein in question, or (if a structure does not exist) knowledge about protein disorder, in the identified region might help to remove false negatives, as LIRs usually lie in intrinsically unstructured regions of the protein.

Caution is advised when probing sequences containing cysteine residues, as those peptides might form disulfide bridges thus causing the spot to produce a false negative. See also Note 2 in [37].

7. Before doing a 20,3 peptide array scan we routinely profile binding to a candidate human LIR-containing protein by GST pulldown assays using in vitro translated, ${ }^{35} \mathrm{~S}$ labeled candidate protein and recombinant, bead-bound GST fusions of LC3A, LC3B, LC3C, GABARAP, GABARAPL1 and GABARAPL2 [41]. 


\section{Acknowledgments}

We are extremely grateful to Ola Rumohr Blingsmo at the Centre for Molecular Medicine

Norway, NCMM - Administration and Core Facilities (NCMM ADMIN), Faculty of

Medicine, University of Oslo for advice and synthesizing the peptide arrays. The technical assistance of Gry Evjen is greatly appreciated. This work was funded by grants from the FRIBIO and FRIBIOMED programs of the Norwegian Research Council (grant numbers 196898 and 214448), and the Norwegian Cancer Society (grant number 71043-PR-20060320) to T.J.

\section{References}

1. Mizushima N, Komatsu M (2011) Autophagy: renovation of cells and tissues. Cell 147:728741

2. Johansen T, Lamark T (2011) Selective autophagy mediated by autophagic adapter proteins. Autophagy 7:279-296

3. Khaminets A, Behl C, Dikic I (2016) Ubiquitin-Dependent And Independent Signals In Selective Autophagy. Trends Cell Biol 26:6-16

4. Rogov V, Dotsch V, Johansen T, Kirkin V (2014) Interactions between autophagy receptors and ubiquitin-like proteins form the molecular basis for selective autophagy. Mol Cell 53:167178

5. Stolz A, Ernst A, Dikic I (2014) Cargo recognition and trafficking in selective autophagy. Nat Cell Biol 16:495-501

6. Pankiv S, Clausen TH, Lamark T, Brech A, Bruun JA, Outzen H, Overvatn A, Bjorkoy G, Johansen T (2007) p62/SQSTM1 binds directly to Atg8/LC3 to facilitate degradation of ubiquitinated protein aggregates by autophagy. J Biol Chem 282:24131-24145

7. Kirkin V, Lamark T, Sou YS, Bjorkoy G, Nunn JL, Bruun JA, Shvets E, Mcewan DG, Clausen TH, Wild P, Bilusic I, Theurillat JP, Overvatn A, Ishii T, Elazar Z, Komatsu M, Dikic I, Johansen T (2009) A role for NBR1 in autophagosomal degradation of ubiquitinated substrates. Mol Cell 33:505-516

8. Wild P, Farhan H, Mcewan DG, Wagner S, Rogov VV, Brady NR, Richter B, Korac J, Waidmann O, Choudhary C, Dotsch V, Bumann D, Dikic I (2011) Phosphorylation of the autophagy receptor optineurin restricts Salmonella growth. Science 333:228-233

9. Thurston TL, Ryzhakov G, Bloor S, Von Muhlinen N, Randow F (2009) The TBK1 adaptor and autophagy receptor NDP52 restricts the proliferation of ubiquitin-coated bacteria. Nat Immunol 10:1215-1221

10. Newman AC, Scholefield CL, Kemp AJ, Newman M, Mciver EG, Kamal A, Wilkinson S (2012) TBK1 kinase addiction in lung cancer cells is mediated via autophagy of Tax1bp1/Ndp52 and non-canonical NF-kappaB signalling. PloS one 7:e50672

11. Lu K, Psakhye I, Jentsch S (2014) Autophagic clearance of polyQ proteins mediated by ubiquitin-Atg8 adaptors of the conserved CUET protein family. Cell 158:549-563 
12. Thurston TL, Wandel MP, Von Muhlinen N, Foeglein A, Randow F (2012) Galectin 8 targets damaged vesicles for autophagy to defend cells against bacterial invasion. Nature 482:414-418

13. Mandell MA, Jain A, Arko-Mensah J, Chauhan S, Kimura T, Dinkins C, Silvestri G, Munch J, Kirchhoff F, Simonsen A, Wei Y, Levine B, Johansen T, Deretic V (2014) TRIM proteins regulate autophagy and can target autophagic substrates by direct recognition. Dev Cell 30:394-409

14. Lynch-Day MA, Klionsky DJ (2010) The Cvt pathway as a model for selective autophagy. FEBS Lett 584:1359-1366

15. Suzuki K, Kondo C, Morimoto M, Ohsumi Y (2010) Selective transport of alpha-mannosidase by autophagic pathways: identification of a novel receptor, Atg34p. J Biol Chem 285:3001930025

16. Mancias JD, Pontano Vaites L, Nissim S, Biancur DE, Kim AJ, Wang X, Liu Y, Goessling W, Kimmelman AC, Harper JW (2015) Ferritinophagy via NCOA4 is required for erythropoiesis and is regulated by iron dependent HERC2-mediated proteolysis. Elife 4

17. Novak I, Kirkin V, Mcewan DG, Zhang J, Wild P, Rozenknop A, Rogov V, Lohr F, Popovic D, Occhipinti A, Reichert AS, Terzic J, Dotsch V, Ney PA, Dikic I (2010) Nix is a selective autophagy receptor for mitochondrial clearance. EMBO Rep 11:45-51

18. Liu L, Feng D, Chen G, Chen M, Zheng Q, Song P, Ma Q, Zhu C, Wang R, Qi W, Huang L, Xue P, Li B, Wang X, Jin H, Wang J, Yang F, Liu P, Zhu Y, Sui S, Chen Q (2012) Mitochondrial outer-membrane protein FUNDC1 mediates hypoxia-induced mitophagy in mammalian cells. Nat Cell Biol 14:177-185

19. Bhujabal Z, Birgisdottir AB, Sjottem E, Brenne HB, Overvatn A, Habisov S, Kirkin V, Lamark T, Johansen T (2017) FKBP8 recruits LC3A to mediate Parkin-independent mitophagy. EMBO Rep

20. Khaminets A, Heinrich T, Mari M, Grumati P, Huebner AK, Akutsu M, Liebmann L, Stolz A, Nietzsche S, Koch N, Mauthe M, Katona I, Qualmann B, Weis J, Reggiori F, Kurth I, Hubner CA, Dikic I (2015) Regulation of endoplasmic reticulum turnover by selective autophagy. Nature 522:354-358

21. Birgisdottir AB, Lamark T, Johansen T (2013) The LIR motif - crucial for selective autophagy. J Cell Sci 126:3237-3247

22. Noda NN, Ohsumi Y, Inagaki F (2010) Atg8-family interacting motif crucial for selective autophagy. FEBS Lett 584:1379-1385

23. Mizushima N, Yoshimori T, Ohsumi Y (2011) The role of Atg proteins in autophagosome formation. Annu Rev Cell Dev Biol 27:107-132

24. Alemu EA, Lamark T, Torgersen KM, Birgisdottir AB, Larsen KB, Jain A, Olsvik H, Overvatn A, Kirkin V, Johansen T (2012) ATG8 Family Proteins Act as Scaffolds for Assembly of the ULK Complex: SEQUENCE REQUIREMENTS FOR LC3-INTERACTING REGION (LIR) MOTIFS. J Biol Chem 287:39275-39290

25. Fu MM, Holzbaur EL (2014) Integrated regulation of motor-driven organelle transport by scaffolding proteins. Trends Cell Biol 24:564-574

26. Olsvik HL, Lamark T, Takagi K, Larsen KB, Evjen G, Overvatn A, Mizushima T, Johansen T (2015) FYCO1 Contains a C-terminally Extended, LC3A/B-preferring LC3-interacting Region (LIR) Motif Required for Efficient Maturation of Autophagosomes during Basal Autophagy. J Biol Chem 290:29361-29374

27. Pankiv S, Alemu EA, Brech A, Bruun JA, Lamark T, Overvatn A, Bjorkoy G, Johansen T (2010) FYCO1 is a Rab7 effector that binds to LC3 and PI3P to mediate microtubule plus end-directed vesicle transport. J Cell Biol 188:253-269

28. Popovic D, Akutsu M, Novak I, Harper JW, Behrends C, Dikic I (2012) Rab GTPaseActivating Proteins in Autophagy: Regulation of Endocytic and Autophagy Pathways by Direct Binding to Human ATG8 Modifiers. Mol Cell Biol 32:1733-1744

29. Mcewan DG, Popovic D, Gubas A, Terawaki S, Suzuki H, Stadel D, Coxon FP, Miranda De Stegmann D, Bhogaraju S, Maddi K, Kirchof A, Gatti E, Helfrich MH, Wakatsuki S, Behrends C, Pierre P, Dikic I (2015) PLEKHM1 Regulates Autophagosome-Lysosome Fusion through HOPS Complex and LC3/GABARAP Proteins. Mol Cell 57:39-54 
30. Genau HM, Huber J, Baschieri F, Akutsu M, Dotsch V, Farhan H, Rogov V, Behrends C (2015) CUL3-KBTBD6/KBTBD7 Ubiquitin Ligase Cooperates with GABARAP Proteins to Spatially Restrict TIAM1-RAC1 Signaling. Mol Cell 57:995-1010

31. Kalvari I, Tsompanis S, Mulakkal NC, Osgood R, Johansen T, Nezis IP, Promponas VJ (2014) iLIR: A web resource for prediction of Atg8-family interacting proteins. Autophagy 10:913-925

32. Von Muhlinen N, Akutsu M, Ravenhill BJ, Foeglein A, Bloor S, Rutherford TJ, Freund SM, Komander D, Randow F (2012) LC3C, bound selectively by a noncanonical LIR motif in NDP52, is required for antibacterial autophagy. Mol Cell 48:329-342

33. Ma P, Schwarten M, Schneider L, Boeske A, Henke N, Lisak D, Weber S, Mohrluder J, Stoldt M, Strodel B, Methner A, Hoffmann S, Weiergraber OH, Willbold D (2013) Interaction of Bcl-2 with the autophagy-related GABAA receptor-associated protein (GABARAP): biophysical characterization and functional implications. J Biol Chem 288:37204-37215

34. Kaufmann A, Beier V, Franquelim HG, Wollert T (2014) Molecular mechanism of autophagic membrane-scaffold assembly and disassembly. Cell 156:469-481

35. Habisov S, Huber J, Ichimura Y, Akutsu M, Rogova N, Loehr F, Mcewan DG, Johansen T, Dikic I, Doetsch V, Komatsu M, Rogov VV, Kirkin V (2016) Structural and Functional Analysis of a Novel Interaction Motif within UFM1-activating Enzyme 5 (UBA5) Required for Binding to Ubiquitin-like Proteins and Ufmylation. J Biol Chem 291:9025-9041

36. Rogov VV, Stolz A, Ravichandran AC, Rios-Szwed DO, Suzuki H, Kniss A, Lohr F, Wakatsuki S, Dotsch V, Dikic I, Dobson RC, Mcewan DG (2017) Structural and functional analysis of the GABARAP interaction motif (GIM). EMBO Rep 18:1382-1396

37. Frank R, Overwin H (1996) SPOT synthesis. Epitope analysis with arrays of synthetic peptides prepared on cellulose membranes. Methods Mol Biol 66:149-169

38. Kramer A, Schneider-Mergener J (1998) Synthesis and screening of peptide libraries on continuous cellulose membrane supports. Methods Mol Biol 87:25-39

39. Lystad AH, Ichimura Y, Takagi K, Yang Y, Pankiv S, Kanegae Y, Kageyama S, Suzuki M, Saito I, Mizushima T, Komatsu M, Simonsen A (2014) Structural determinants in GABARAP required for the selective binding and recruitment of ALFY to LC3B-positive structures. EMBO Rep 15:557-565

40. Skytte Rasmussen M, Mouilleron S, Kumar Shrestha B, Wirth M, Lee R, Bowitz Larsen K, Abudu Princely Y, O'reilly N, Sjottem E, Tooze SA, Lamark T, Johansen T (2017) ATG4B contains a C-terminal LIR motif important for binding and efficient cleavage of mammalian orthologs of yeast Atg8. Autophagy 13:834-853

41. Johansen T, Birgisdottir AB, Huber J, Kniss A, Dotsch V, Kirkin V, Rogov VV (2017) Methods for Studying Interactions Between Atg8/LC3/GABARAP and LIR-Containing Proteins. Methods Enzymol 587:143-169 


\section{FIGURE LEGENDS}

Fig. 1 Mapping of a single LIR motif in FKBP8 using a peptide array of 20-mer peptides covering full-length FKBP8 of 412 amino acids. The array was probed with $1 \mu \mathrm{g} / \mathrm{ml}$ of GSTGABARAP for $2 \mathrm{hrs}$, and binding to GST-GABARAP detected with anti-GST antibody using chemiluminescence. (A) Schematic drawing of the domain architecture of human FKBP8. (B) Image of the developed film of a 20,3 peptide array containing 20-mer peptides spotted on a 540 grid membrane with 18 rows of 30 spots in the horizontal direction. Each peptide was shifted three amino acids relative to the previous peptide. Only the part of the membrane containing peptides derived from the sequence of FKBP8 is shown. Five positive spots are seen in position 4 to 8 in the array. $(\mathbf{C})$ The sequence of the region covered by five peptides corresponding to the positive spots in (B). The identified consensus LIR motif is indicated in grey and the relevant amino acid positions shown. The extent of each peptide is indicated with horizontal bars below the sequence. (D) The sequences of the five GABARAP-interacting peptides are shown. (E) Schematic drawing of the membrane with the position of spotted peptides shown and the positive spots indicated as black dots. L, LIR motif; EEE, glutamaterich domain; P; proline-rich sequence; PPIase, peptidyl-prolyl cis-trans isomerase domain; TPR, tetratricopeptide repeats: CaM, calmodulin binding domain and TM, transmembrane domain. (Adapted from ref. [19]).

Fig. 2 Peptide array with 20-mer peptides containing identified LIR motifs from ULK1, ULK2, ATG4B and FYCO1 (synthesized in duplicates marked 1 and 2) were examined for their binding preference towards GST-tagged ATG8 homologues LC3A, LC3B, GABARAP and GABARAPL2. The peptide arrays were probed with $1 \mu \mathrm{g} / \mathrm{ml}$ of the different GST-ATG8 
fusion proteins for $2 \mathrm{~h}$, and binding was detected with anti-GST antibodies. (Adapted from ref. [40]).

Fig. 3 A two-dimensional peptide array scan analyzing the effects of single amino acid substitutions at all positions of the indicated 18-mer peptides from FYCO1 (amino acids 1276-1293). Each position of the 18-mer peptides was replaced with all 20 amino acids. The peptide array was probed with $1 \mu \mathrm{g} / \mathrm{ml}$ GST-LC3B for $2 \mathrm{~h}$, and binding to GST-LC3B was detected with anti-GST antibodies. This analysis allowed the identification of the residues EL C-terminal to the core LIR motif as crucial for binding and the show that functional LIR is likely at least 13 amino acids. At the aromatic position binding in HP1, only F/W/Y are tolerated. At the hydrophobic position binding in HP2, only I/L/V are tolerated. Basic residues $\mathrm{K}$ and $\mathrm{R}$ and secondary structure disrupting $\mathrm{G}$ and $\mathrm{P}$ residues are not tolerated in the core LIR. (Adapted from ref. [26]). 


\section{FIGURE 1}

A

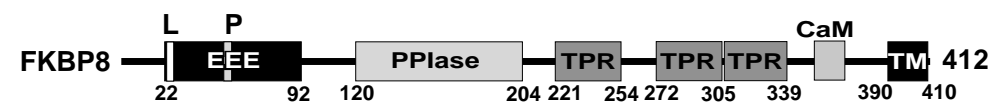

B

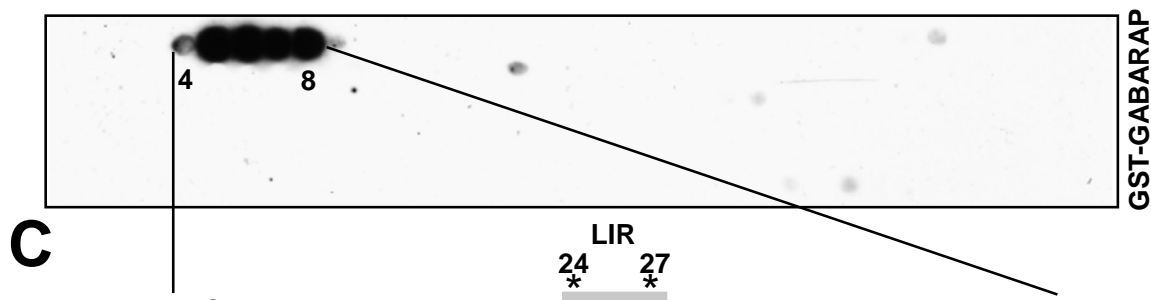

FKBP8 10 P-S-A-P-L-P-A-G-V-P-P-L-E-D-F-E-V-L-D-G-V-E-D-A-E-G-E-E-E-E-E-E 41

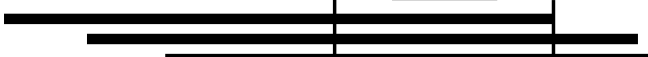

D

peptide no A4 P-S-A-P-L-P-A-G-V-P-P-L-E-D-F-E-V-L-D-G

peptide no A5 P-L-P-A-G-V-P-P-L-E-D-F-E-V-L-D-G-V-E-D

peptide no A6 A-G-V-P-P-L-E-D-F-E-V-L-D-G-V-E-D-A-E-G

peptide no A7 P-P-L-E-D-F-E-V-L-D-G-V-E-D-A-E-G-E-E-E

peptide no A8 E-D-F-E-V-L-D-G-V-E-D-A-E-G-E-E-E-E-E-E

$\mathbf{E}$

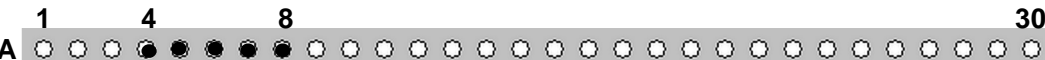

B000000000000000000000000000000

C000000000000000000000000000000

D000000000000000000000000000000

E000000000000000000000000000000 


\section{FIGURE 2}

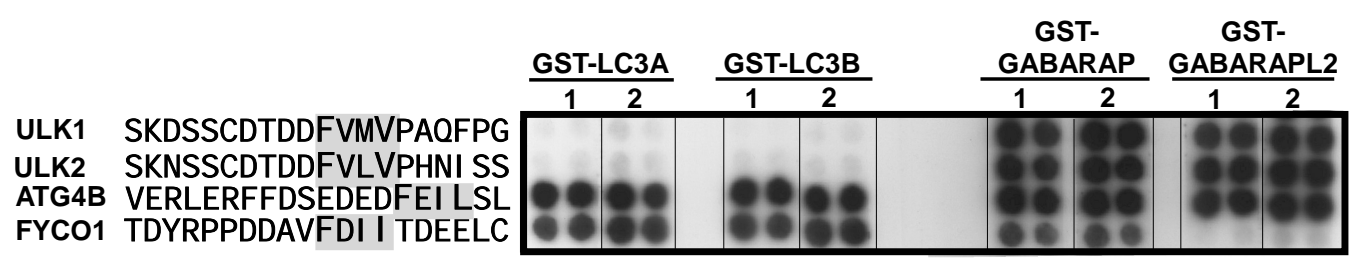




\section{FIGURE 3}

FYCO1 (aa 1276-1293)

LC3B binding

1276

$1280 \quad 1283$

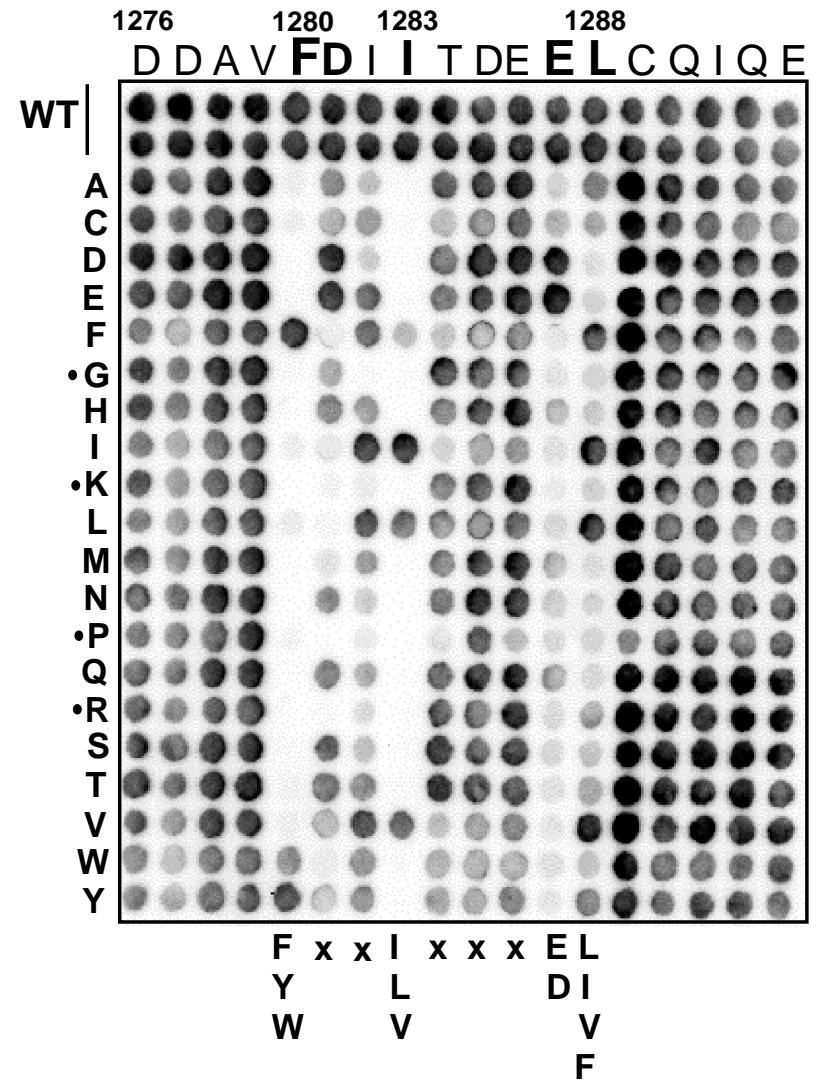

